\title{
Detection and correction of prescription errors by an emergency department pharmacy service
}

\author{
Philip Stasiak, MD*; Marc Afilalo, MD ${ }^{\dagger}$; Tanya Castelino, $\mathrm{BSc}^{\ddagger}$; Xiaoqing Xue, $\mathrm{MSc}^{\dagger}$; \\ Antoinette Colacone, BSc-CCRA ${ }^{\dagger}$; Nathalie Soucy, $\mathrm{PhD}^{\dagger}$; Jerrald Dankoff, $\mathrm{MD}^{\dagger}$
}

\section{ABSTRACT}

Objectives: Emergency departments (EDs) are recognized as a high-risk setting for prescription errors. Pharmacist involvement may be important in reviewing prescriptions to identify and correct errors. The objectives of this study were to describe the frequency and type of prescription errors detected by pharmacists in EDs, determine the proportion of errors that could be corrected, and identify factors associated with prescription errors.

Methods: This prospective observational study was conducted in a tertiary care teaching ED on 25 consecutive weekdays. Pharmacists reviewed all documented prescriptions and flagged and corrected errors for patients in the ED. We collected information on patient demographics, details on prescription errors, and the pharmacists' recommendations.

Results: A total of 3,136 ED prescriptions were reviewed. The proportion of prescriptions in which a pharmacist identified an error was $3.2 \%$ (99 of 3,136; 95\% confidence interval $[\mathrm{Cl}] 2.5-3.8)$. The types of identified errors were wrong dose (28 of 99, 28.3\%), incomplete prescription (27 of $99,27.3 \%$ ), wrong frequency (15 of $99,15.2 \%)$, wrong drug (11 of $99,11.1 \%)$, wrong route ( 1 of $99,1.0 \%$ ), and other (17 of $99,17.2 \%)$. The pharmacy service intervened and corrected 78 (78 of $99,78.8 \%$ ) errors. Factors associated with prescription errors were patient age over 65 (odds ratio [OR] 2.34; 95\% Cl 1.32-4.13), prescriptions with more than one medication (OR 5.03; 95\% Cl 2.54-9.96), and those written by emergency medicine residents compared to attending emergency physicians (OR 2.21, 95\% Cl 1.184.14).

Conclusions: Pharmacists in a tertiary ED are able to correct the majority of prescriptions in which they find errors. Errors are more likely to be identified in prescriptions written for older patients, those containing multiple medication orders, and those prescribed by emergency residents.

\section{RÉSUMÉ}

Objectifs: C'est connu, les services d'urgence (SU) sont un lieu où le risque d'erreurs de prescription est élevé. Alors, les pharmaciens peuvent jouer un rôle important dans la révision des prescriptions dans le but de repérer et de corriger les erreurs. L'étude avait pour objectifs de décrire la fréquence des erreurs de prescription décelées par les pharmaciens au SU et leur type, de déterminer la proportion des erreurs susceptibles de correction et de cerner les facteurs associés aux erreurs de prescription.

Méthodes: II s'agit d'une étude d'observation, prospective, menée dans un SU d'enseignement, de soins tertiaires, pendant 25 semaines consécutives. Les pharmaciens ont révisé toutes les prescriptions documentées, rédigées au $\mathrm{SU}$, puis marqué et corrigé les erreurs. Il y a eu une collecte de renseignements sur les données démographiques des patients, sur les éléments concernant les erreurs de prescription ainsi que sur les recommandations des pharmaciens.

Résultats: Au total, 3136 prescriptions rédigées au SU ont été révisées. La proportion de prescriptions comportant une erreur décelée par les pharmaciens s'est élevée à 3.2\% (99 sur 3136; intervalle de confiance [IC] à 95\%: 2.5-3.8). Les types d'erreur relevés étaient la mauvaise dose (28 sur 99; $28.3 \%$ ), une prescription incomplète (27 sur $99 ; 27.3 \%$ ), la mauvaise fréquence (15 sur $99 ; 15.2 \%$ ), le mauvais médicament (11 sur $99 ; 11.1 \%$ ), la mauvaise voie d'administration ( 1 sur $99,1.0 \%$ ) et le reste (17 sur $99 ; 17.2 \%)$. Le service de pharmacie a corrigé 78 erreurs (78 sur 99; 78.8\%). Les facteurs associés aux erreurs de prescription comprenaient

From the *McGill Emergency Medicine Residency Program; †Emergency Department, Sir Mortimer B. Davis Jewish General Hospital; and $\neq$ Faculty of Medicine, McGill University, Montreal, QC.

Abstract presented at the Canadian Association of Emergency Physicians Conference, Calgary, AB, June 2009; Association des médecins d'urgence du Québec Conference, Victoriaville, QC, November 2009; and International Conference on Emergency Medicine, Valencia, Spain, September 2009.

Correspondence to: Dr. Philip Stasiak, Emergency Department Room D-027, Jewish General Hospital, 3755 Côte-Sainte-Catherine Road, Montréal, OC H3T 1E2; philip.stasiak@mail.mcgill.ca.

This article has been peer reviewed. 
un âge supérieur à 65 ans chez les patients (risque relatif approché [RRA]: 2.34; IC à 95\%: 1.32-4.13), la prescription de plus d'un médicament (RRA: 5.03; IC à 95\%: 2.54-9.96) et la rédaction de prescriptions par les résidents en médecine d'urgence comparativement aux urgentologues traitants (RRA: 2.21; IC à 95\%: 1.18-4.14).

Conclusions: Les pharmaciens dans un SU de soins tertiaires sont capables de corriger la plupart des erreurs décelées dans les prescriptions. Le risque d'erreur est plus élevé dans les prescriptions concernant les patients âgés, celles comptant plusieurs médicaments et celles rédigées par les résidents en médecine d'urgence.

Keywords: adverse drug events, emergency department, patient safety, pharmacist, prescription errors
Emergency departments (EDs) are high-risk settings for medical errors. ${ }^{1}$ Emergency physicians provide episodic care to patients they typically do not have a previous relationship with in urgent and emergent situations and in a suboptimal environment. Factors such as overcrowding, ${ }^{2}$ staffing shortages, ${ }^{3,4}$ frequent interruptions, and handovers in care $^{4}$ contribute to making the ED a high-risk setting.

Medication errors are one of the most common types of ED errors. ${ }^{3,5,6}$ Most medication errors are related to one or more of "five wrongs" involved in the act of prescribing: wrong drug, wrong dose, wrong route, wrong time, and wrong patient. ${ }^{7,8}$ Certain types of drugs, such as analgesics, antibiotics, sedatives, and cardiovascular drugs, have been associated with more frequent prescription errors compared to other drug classes. ${ }^{3,7,9}$ In addition, prescribing to more complex and acutely ill patients at nighttime, on weekends, or by trainees has been associated with more frequent errors. ${ }^{7,9}$

Previous studies have estimated the proportion of prescriptions with errors in adult inpatients and in ED patient populations at between 0.4 and $6.3 \% .^{5,10-13}$ Other studies in the pediatric ED setting have found that as many as $10 \%$ of medical charts contained prescription errors. ${ }^{9} 14,15$ However, these studies were conducted either in inpatient or pediatric patient populations, used retrospective designs, or were based on a short period of data collection.

There is increasing evidence for the benefits of employing a pharmacist in the $\mathrm{ED}^{16-21}$ and the important role pharmacists can play in identifying and decreasing prescription errors. ${ }^{10-12,20-23}$ To date, no studies have described the frequency of, classification of, and risk factors for prescription errors in Canadian EDs. ${ }^{6}$

The objectives of this study were to describe the frequency and type of prescription errors detected by pharmacists in EDs, determine the proportion of errors that could be corrected, and identify factors associated with prescription errors.

\section{METHODS}

\section{Study design and setting}

This was a single-centre, prospective, observational study conducted in the ED of the Jewish General Hospital, a tertiary care teaching hospital in Montreal. Our ED serves an adult patient population and had an annual census of 66,000 in 2008. This study was approved by the Research Ethics Committee of the Jewish General Hospital.

At the time of the study, the daily operations of the ED were divided into three clinical shifts composed of a day shift (08:00-16:00), an evening shift (16:0024:00), and a night shift (24:00-08:00). Two pharmacists and one pharmacy technician participated in this study. One pharmacist and one pharmacy technician were on site on weekdays from 08:00 to 16:00, whereas one technician was on site between 16:00 and 21:00 on weekday evenings. No pharmacy service was available between 21:00 and 08:00 on weekdays or at any time on weekends and holidays. The pharmacists' duties included supplying medications that were not available in the ED and reviewing all ED prescriptions, including transcribed verbal orders. The pharmacy service was informed of the objectives of this study; however, their duties were not altered by participation.

We included all prescriptions that were written or verbally transmitted and transcribed in our ED for 25 consecutive weekdays between November 17 and December 19, 2008. We excluded discharge prescriptions and prescriptions written during weekends.

Medication orders in our ED were written by attending and resident physicians on standardized paper forms located in the patients' charts and were picked up by nurses. Nurses gave a copy of the 
recorded prescription to the pharmacist for review for the purposes of this study, obtained the medication, and then carried out the order. Physicians communicated verbal orders directly to nurses. Nurses then recorded verbal orders on a prescription sheet and carried them out. Verbal orders were countersigned by the physician at a subsequent point in time. The copy of transmitted verbal orders was given to the pharmacist for review.

Every morning, the incoming pharmacist reviewed all retained copies of prescriptions written in the ED during the previous evening, night, and early morning. The pharmacist identified prescriptions that were erroneous and attempted to correct them by speaking with the physician if the patient was still in the ED and the medication had not yet been dispensed or was an ongoing prescription.

Prescriptions were deemed to have an error if the on-duty ED pharmacist recommended making any addition or modification to them. The pharmacist contacted the physician to clarify any uncertainties about the determination of the appropriateness of or indication for the prescription. For example, if a penicillin-allergic patient was prescribed ticarcillinclavulanate, the pharmacist would contact the physician and recommend an alternate antibiotic. When the pharmacy technician suspected an error, he or she reported this to the pharmacist as soon as possible. The pharmacist then reviewed the prescription and contacted the physician regarding any errors.

Pharmacists initially classified errors at the time of reviewing the prescriptions. Subsequently, two other researchers (N.S. and T.C.) reviewed all cases and, for cases with disagreement, determined the error classification through discussion and consensus. Patient charts and pharmacy records were used during this process. We classified errors into six categories: 1) wrong drug; 2) wrong dose; 3) wrong frequency; 4) wrong route; 5) incomplete prescriptions; and 6) others. The wrong drug category included patient allergy to a prescribed drug or an inappropriate drug for the disease. Wrong dose, wrong frequency, and wrong route were determined according to usual hospital and pharmacy practice. Incomplete prescription included missing information related to route, dose, or medication frequency. The other category included redundant medications, missing signatures, nonformulary medications, illegible prescriptions, and conflicting orders.

\section{Data collection}

For the purposes of data collection, the pharmacist attached a note to the copy of all prescriptions in which one or more errors were found. These copies were collected from the pharmacy service on a daily basis. The pharmacist's note provided information on the category of error identified, the nature of the pharmacist's recommendation, and whether or not the recommendation was followed. In addition, the prescriber, the time and date of the prescription, and the number of medication orders written on the prescription were recorded. Prescribers included attending emergency physicians, emergency residents, and specialist consultants (staff and residents). Information on the patients' age, gender, triage code, chief complaint, main diagnosis, mode of arrival, and stretcher use was obtained through our ED electronic database.

\section{Outcome measures and data analysis}

The primary outcome was the number and proportion of prescription errors identified by the pharmacy service. Secondary outcomes were the error categorization, proportion of errors corrected, and factors associated with prescription errors.

Descriptive statistics such as means (with standard deviations), medians (with interquartile ranges), and proportions (with $95 \%$ confidence intervals [CIs]) were used to describe the baseline characteristics of enrolled patients. We conducted univariate analyses using $t$ tests, chi-square tests, or Fisher exact tests as required to determine whether there were any associations between the presence of one or more prescription errors and the following variables defined a priori: age $>65$ years, the number of prescribed medications (one medication, $\geq 2$ medications), prescriber type (attending emergency physician, emergency resident, specialist consultant [attending physicians and residents]), arrival by ambulance (yes, no), Canadian Triage Acuity Scale (CTAS) triage level (grouped into CTAS 1 and 2, CTAS 3, and CTAS 4 and 5), and shift (day, evening, and night) during which the prescription was written. Those variables that were associated with the occurrence of one or more prescription errors based on the results of univariate analyses $(p \leq 0.10)$ were entered into a logistic regression model with a generalized estimating equation (GEE) to identify variables independently 
associated with prescription errors. The GEE analysis was used to account for the clustering effect of prescriptions nested within patient visits. The results from the logistic regression analysis are presented as odds ratios (ORs) and 95\% CIs. All data management and statistical analyses were conducted using $S A S$ version 9.2 (SAS Institute Inc., Cary, NC).

\section{RESULTS}

The study sample was made up of 1,088 ED visits from 1,025 unique patients. Table 1 shows the baseline characteristics of patients and ED visits in the study. The median age of patients was 75.3 years. The median ED length of stay was 31.8 hours. Forty-eight percent of ED visits occurred during the daytime, and $47 \%$ led to a hospitalization. These visits generated 3,136 ED prescriptions containing 11,313 medication orders that were reviewed by the ED pharmacy service.

\begin{tabular}{|c|c|}
\hline Characteristic & \\
\hline \multicolumn{2}{|l|}{ Patient } \\
\hline Median age, yr (interquartile range) & $75.3(60-84)$ \\
\hline Female gender, $n(\%)$ & $576(53.0)$ \\
\hline \multicolumn{2}{|l|}{ Visit } \\
\hline \multicolumn{2}{|l|}{ CTAS triage category, $n(\%)$} \\
\hline 1 & $4(0.4)$ \\
\hline 2 & $348(32.0)$ \\
\hline 3 & $592(54.4)$ \\
\hline 4 & $141(13.0)$ \\
\hline 5 & $3(0.3)$ \\
\hline \multicolumn{2}{|l|}{ Mode of arrival, $n(\%)$} \\
\hline By ambulance & $509(46.8)$ \\
\hline Walk-in & $579(53.2)$ \\
\hline \multicolumn{2}{|l|}{ Shift of ED visit, $n(\%)$} \\
\hline Day shift & $517(47.5)$ \\
\hline Evening shift & $393(36.1)$ \\
\hline Night shift & $178(16.4)$ \\
\hline \multicolumn{2}{|l|}{ Most common chief complaints } \\
\hline Abdominal pain & $119(10.9)$ \\
\hline Chest pain & 119 (10.9) \\
\hline Shortness of breath & $111(10.2)$ \\
\hline Generalized weakness & $60(5.5)$ \\
\hline Nausea or vomiting & $49(4.5)$ \\
\hline \multicolumn{2}{|l|}{ Disposition, $n(\%)$} \\
\hline Hospitalized & $509(46.8)$ \\
\hline Nonhospitalized & $579(53.2)$ \\
\hline $\begin{array}{l}\text { Median ED length of stay, hr } \\
\text { (interquartile range) }\end{array}$ & $31.8(19.5-55.7)$ \\
\hline
\end{tabular}

Among the prescriptions reviewed, 99 errors (99 of 3,136; 3.2\%; 95\% CI 2.5-3.8) were identified among 81 prescriptions (see Appendix, available at http:// www.cjem-online.ca), illustrating that some prescriptions contained multiple errors. Pharmacists corrected $78(78.8 \%)$ of the errors identified.

Figure 1 shows the types of prescription errors identified and the proportions corrected within each category. Among 99 errors, 28 (28.3\%) were due to the wrong dose, followed by incomplete prescription (27; $27.3 \%)$, other $(17 ; 17.2 \%)$, wrong frequency (15; $15.2 \%)$, wrong drug $(11 ; 11.1 \%)$, and wrong route of administration $(1 ; 1.0 \%)$.

Table 2 illustrates prescription characteristics and includes the proportion of errors classified according to the shift that the prescriptions were written in, categorized by type of prescriber, patient age, and number of medication orders on the prescription.

On univariate analyses, four factors were found to be associated with the presence of prescription errors, at a significance level of $\leq 0.1$ : patient age, number of prescribed medications, prescriber type, and means of arrival. Table 3 shows the factors independently associated with prescription errors on multivariable analysis. Prescriptions written for patients over the age of 65 years (OR 2.34; 95\% CI 1.32-4.13), those that included more than one medication order (OR 5.03; 95\% CI 2.54-9.96), and those written by emergency residents (OR 2.21; 95\% CI 1.18-4.14) compared to attending emergency physicians were all found to have a higher odds of a prescription error.

Table 4 provides a categorization of prescription errors by type of medication. The most frequent drug classes associated with prescription errors were cardiovascular drugs ( 21 of $99 ; 21.2 \%$ ), neurologic drugs (10 of $99 ; 10.1 \%$ ), and inhaled medications (9 of 99; $9.1 \%$ ). The most frequent drugs with errors were diltiazem (7 of $99 ; 7.1 \%$ of all errors), acetylcysteine(4 of $99 ; 4.0 \%$ ), and carbidopa-levodopa (4 of 99; 4.0\%).

We found no errors among the 142 verbal orders that were transcribed and reviewed. The three most common verbal orders were for intravenous fluids (33 of $142 ; 23.2 \%$ ), acetaminophen ( 23 of $142 ; 16.2 \%)$, and morphine (16 of $142 ; 11.3 \%)$.

\section{DISCUSSION}

This study describes the frequency and types of prescription errors detected and corrected by the 


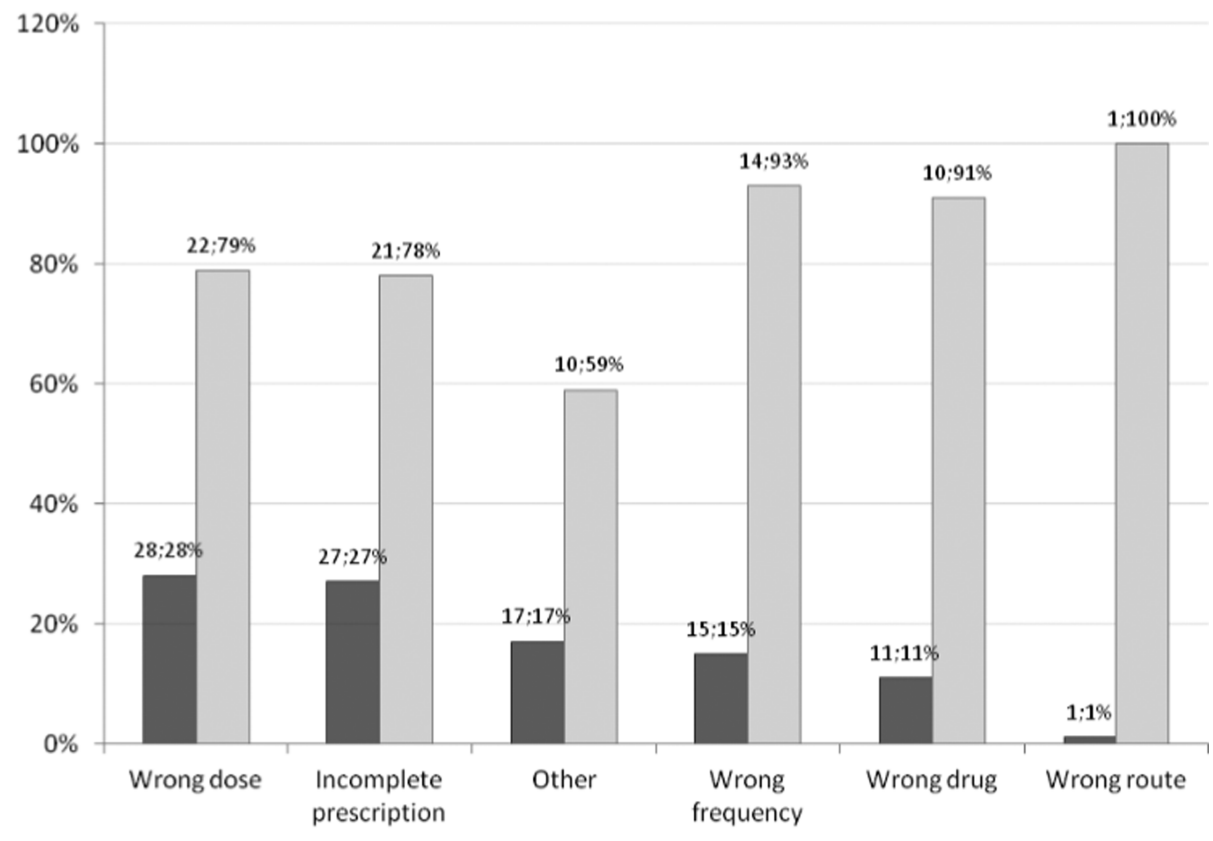

Figure 1. Types of prescription errors and proportions corrected $(n ; \%)$.

Number and percentage of type of error identified among 99 errors

$\square$ Number and percentage of error corrected within each type of error

pharmacy service in a tertiary ED. Through multivariable analysis, we identified factors that were associated with prescription errors in our ED. Pharmacists identified 99 (3.2\%) errors in 3,136 ED prescriptions, of which $78.8 \%$ were corrected through their intervention.
The proportion of errors we found is within the range reported in other studies., ${ }^{510-12,24,25}$ Variability in this estimate is likely due, at least in part, to the different methodologies and settings in which other studies were conducted. When describing the types of prescription error, Kozer and colleagues found a

\begin{tabular}{|c|c|c|c|}
\hline Characteristic & Error $(n=81)$ & No error $(n=3,055)$ & Total $(n=3,136)$ \\
\hline \multicolumn{4}{|c|}{ Shift during which the prescription was written, $n(\%)$} \\
\hline Day shift & $12(1.5)$ & 809 (98.5) & 821 \\
\hline Evening shift & 37 (3.8) & $929(96.2)$ & 966 \\
\hline Night shift & $16(2.5)$ & $625(97.5)$ & 641 \\
\hline Shift information missing & $16(2.5)$ & $692(97.7)$ & 708 \\
\hline \multicolumn{4}{|l|}{ Type of prescriber, $n(\%)$} \\
\hline EM attending staff & $29(2.1)$ & $1,328(97.9)$ & 1,357 \\
\hline EM residents & $16(5.6)$ & $268(94.4)$ & 284 \\
\hline Specialty consultants and residents & $36(2.4)$ & $1,453(97.6)$ & 1,489 \\
\hline Prescriber type missing & $0(0)$ & $6(100)$ & 6 \\
\hline \multicolumn{4}{|l|}{ Patient age, $n(\%)$} \\
\hline$\geq 65$ years old & $67(3.2)$ & $2,059(96.8)$ & 2,126 \\
\hline$<65$ years old & $14(1.4)$ & $996(98.6)$ & 1,010 \\
\hline \multicolumn{4}{|c|}{ Number of medication orders per prescription, $n(\%)$} \\
\hline One medication order & $9(0.8)$ & $1,188(99.2)$ & 1,197 \\
\hline$\geq 2$ medication orders & $72(3.7)$ & $1,867(96.3)$ & 1,939 \\
\hline
\end{tabular}


Table 3. Factors independently associated with prescription errors on multivariable analysis

\begin{tabular}{llll} 
Factor name & \multicolumn{1}{c}{ Factor level } & Odds ratio & $95 \% \mathrm{Cl}$ \\
Type of prescriber & Emergency medicine residents & 2.21 & $1.18-4.14$ \\
& Specialty staff and residents & 0.84 & $0.51-1.40$ \\
& Emergency attending staff & 1.00 & Reference \\
Age & $\geq 65 \mathrm{yr}$ & 2.34 & $1.32-4.13$ \\
& $<65 \mathrm{yr}$ & 1.00 & Reference \\
Number of medication orders per prescription & 2 or more medications ordered & 5.03 & $2.54-9.96$ \\
& 1 medication ordered & 1.00 & Reference
\end{tabular}

similar pattern in the pediatric setting. ${ }^{9}$ The majority of prescription errors were related to the wrong dose $(49 \%)$ and the wrong frequency $(43 \%)$, whereas the wrong route $(3 \%)$, the wrong drug $(2 \%)$, and the wrong information $(3 \%)$ were far less common. In another study in both adult and pediatric patients, Rothschild and colleagues similarly found drug dose errors $(40 \%)$ to be the most common. ${ }^{13}$

We found three factors that were more likely to be associated with prescription errors: prescriptions written by emergency medicine residents, those written for elderly patients, and those involving multiple medications. The first factor may be a reflection of the complexity for trainees of prescribing in the ED. Emergency medicine residents, when compared to emergency attending physicians, are less familiar with medications, dosages, dosing regimens, and treatments because they have less experience with the breadth of medical problems encountered in the ED. With regard to age, our findings are consistent with other studies showing that physicians are more likely to commit errors when prescribing to the elderly. ${ }^{26-28}$ Elderly

\begin{tabular}{|c|c|}
\hline Medication category & Number of errors $(\%)(N=99)$ \\
\hline Cardiovascular & $21(21.2)$ \\
\hline Neurologic & $10(10.1)$ \\
\hline Inhaled medications & $9(9.1)$ \\
\hline Ophthalmic drops & $8(8.1)$ \\
\hline Vitamins and supplements & $8(8.1)$ \\
\hline Antimicrobials & $7(7.1)$ \\
\hline Gastrointestinal & $6(6.1)$ \\
\hline Diabetes & $6(6.1)$ \\
\hline Psychiatric & $6(6.1)$ \\
\hline Other & $18(18.2)$ \\
\hline
\end{tabular}

patients tend to have more comorbidities and a larger number of coprescribed medications, both of which can result in contraindications and interactions, thus complicating the prescribing process. Finally, and similar to the findings of Patanwala and colleagues, ${ }^{29}$ our finding with regard to multiple medicine prescriptions is explained by the fact that the complexity of prescribing increases as the number of medications ordered increases, thus compounding the potential for error.

Although the pharmacy service was not available 24/ 7 during the study period, this service was able to correct the majority of identified prescription errors. Errors that were not corrected predominantly arose as a result of patients leaving the ED before the pharmacist was able to review the prescription.

Among the 65 prescriptions with errors where the shift was identified, more than half of the errors (37 of 65 ) occurred during the evening. This may have arisen because of pharmacists' availability during the day shift to assist with complicated prescriptions, thus contributing to safer prescribing during these times. The proportion of prescription errors involving cardiovascular and neurologic drugs, vitamins, and supplements may be due to the wide range of formulations available for these agents. Rothschild and colleagues also found that central nervous system drugs and cardiovascular drugs were commonly associated with prescription errors. ${ }^{13}$ Similarly, different brands of supplements may contain varying amounts of compounds and can be confusing for prescribers. Inhalers and ophthalmic solutions can represent unconventional prescriptions, and prescribers unfamiliar with these may be more prone to error..$^{30}$

Very little research has been published on verbal orders. ${ }^{31}$ Although we acknowledge the potential dangers of verbal orders, we found no errors among 
the 142 verbal orders we reviewed. This counterintuitive finding is consistent with those of another study looking at verbal orders in the paediatric inpatient setting, which found a fourfold decreased risk of error associated with verbal orders compared to handwritten orders. ${ }^{32}$ Increased vigilance by the transcribers and the commonality of and resulting familiarity with medications that are typically prescribed verbally may explain these findings.

\section{LIMITATIONS}

This study has limitations that should be considered when interpreting our findings. It was conducted at a single adult tertiary care centre, which limits the generalizability of our findings. Moreover, we relied solely on the pharmacy service for the detection and correction of prescription errors. It is possible that the pharmacy service did not detect all errors that occurred and that some of the apparent errors detected may have arisen from intentional variations on the part of the prescriber. Notes stapled to the prescription indicated an error, but we assumed that no note signified the lack of an error, although some such cases could have represented a missing note. In addition, we may have missed some prescriptions for which copies were accidentally discarded or misplaced. Because of the pharmacy service schedule, we collected data only for a limited period of time during weekdays. Consequently, our results may not reflect the proportion or types of errors occurring on weekends or holidays. We did not study adverse drug reactions, drug interactions, the need for closer monitoring, or the need for antidotes as a result of ED prescriptions; therefore, we are unable to determine the impact of the prescription errors that we identified and did not correct.

\section{CONCLUSIONS}

Pharmacists in a tertiary ED are able to correct the majority of prescriptions in which they found errors. Errors are more likely to be identified in prescriptions written for older patients, those containing multiple medication orders, and those prescribed by emergency residents. Finally, we found no errors in any of the verbal orders we reviewed. A better understanding of the consequences of prescription errors that are not intercepted, as well as of the causes of prescription errors, may lead to further improvements in medication safety in the ED.

Acknowledgements: We would like to acknowledge the hard work and diligence of Talia Klein, Jong Lee, and Noemie Dreksler.

Competing interests: This work was funded by the Canadian Patient Safety Institute.

\section{REFERENCES}

1. Kohn LT, Corrigan J, Donaldson MS, editors. To err is buman: building a safer bealth system. Washington (DC): National Academy Press; 2000.

2. Kulstad EB, Sikka R, Sweis RT, et al. ED overcrowding is associated with an increased frequency of medication errors. Am 7 Emerg Med 2010;28:304-9, doi:10.1016/j.ajem.2008. $\underline{12.014}$.

3. Phillips DP, Bredder CC. Morbidity and mortality from medical errors: an increasingly serious public health problem. Annu Rev Public Health 2002;23:135-50, doi:10. 1146/annurev.publhealth.23.100201.133505.

4. Chisholm CD, Collison EK, Nelson DR, et al. Emergency department workplace interruptions: are emergency physicians "interrupt-driven" and "multitasking"? Acad Emerg Med 2000;7:1239-43, doi:10.1111/j.1553-2712.2000.tb00469.x.

5. Jenkins G, Johnston PE, Patel NR, et al. The epidemiology of medication prescribing errors in the emergency department. AMIA Annu Symp Proc 2006:968.

6. Croskerry P, Shapiro M, Campbell S, et al. Profiles in patient safety: medication errors in the emergency department. Acad Emerg Med 2004;11:289-99, doi:10.1111/j.1553-2712. 2004.tb02214.x.

7. Benjamin DM. Minimizing medication errors: practical pointers for prescribers. 7 Clin Pharmacol 2003;43:751-3.

8. Peth HA. Medication errors in the emergency department, a systems approach to minimizing risk. Emerg Med Clin North Am 2003;21:141-58, doi:10.1016/S0733-8627(02)00085-8.

9. Kozer E, Scolnik D, Macpherson A, et al. Variables associated with medication errors in pediatric emergency medicine. Pediatrics 2002;110:737-42, doi:10.1542/peds.110. $\underline{4.737 .}$

10. Bobb A, Gleason K, Husch M, et al. The epidemiology of prescribing errors: the potential impact of computerized prescriber order entry. Arch Intern Med 2004;164:785-92, doi:10.1001/archinte.164.7.785.

11. Dean B, Schachter M, Vincent C, et al. Prescribing errors in hospital inpatients: their incidence and clinical significance. Qual Saf Health Care 2002;11:340-4, doi:10.1136/qhc. 11.4.340.

12. Lesar TS, Briceland L, Stein DS. Factors related to errors in medication prescribing. 7 Am Med Assoc 1997;277:312-7, doi:10.1001/jama.1997.03540280050033.

13. Rothschild JM, Churchill W, Erickson A, et al. Medication errors recovered by emergency department pharmacists. Ann Emerg Med 2010;55:513-21, doi:10.1016/j.annemergmed. 2009.10.012. 
14. Rinke ML, Moon M, Clark JS, et al. Prescribing errors in a pediatric emergency department. Pediatr Emerg Care 2008; 24:1-8.

15. Taylor BL, Selbst SM, Shah AE. Prescription writing errors in the pediatric emergency department. Pediatr Emerg Care 2005;21:822-7, doi:10.1097/01.pec.0000190239. 04094.72 .

16. Vasileff HM, Whitten LE, Pink JA, et al. The effect on medication errors of pharmacists charting medication in an emergency department. Pharm World Sci 2009;31:373-9, doi:10.1007/s11096-008-9271-y.

17. Clancy CM. Evidence shows cost and patient safety benefits of emergency pharmacists. Am 7 Med Qual 2008;23:231-3, doi: $10.1177 / 1062860608316108$.

18. Case LL, Paparella S. Safety benefits of a clinical pharmacist in the emergency department. 7 Emerg Nurs 2007;33:564-6, doi:10.1016/j.jen.2007.06.008.

19. Lada P, Delgado G. Documentation of pharmacists' interventions in an emergency department and associated cost avoidance. Am 7 Health Syst Pharm 2007;64:63-8, doi:10.2146/ajhp050213.

20. Marconi GP, Claudius I. Impact of an emergency department pharmacy on medication omission and delay. Pediatr Emerg Care 2012;28:30-3, doi:10.1097/PEC.0b013e31823 f232d.

21. Abu-Ramaileh AM, Shane R, Churchill W, et al. Evaluating and classifying pharmacists' quality interventions in the emergency department. Am 7 Health Syst Pharm 2011;68: 2271-5, doi:10.2146/ajhp110004.

22. Brown JN, Barnes CL, Beasley B, et al. Effect of pharmacists on medication errors in an emergency department. Am 7 Health Syst Pharm 2008;65:330-3, doi:10.2146/ajhp 070391.

23. Guy J, Persaud J, Davies E, et al. Drug errors: what role do nurses and pharmacists have in minimizing the risk? 7 Child Health Care 2003;7:277-90, doi:10.1177/1367 4935030074004.
24. Hendey GW, Barth BE, Soliz T. Overnight and postcall errors in medication orders. Acad Emerg Med 2005;12:62934, doi:10.1111/j.1553-2712.2005.tb00919.x.

25. Bizovi KE, Wears R, Lowe RA. Researching quality in emergency medicine. Acad Emerg Med 2002;9:1116-23, doi:10.1111/j.1553-2712.2002.tb01565.x.

26. Lam P, Elliott RA, George J. Impact of a self-administration of medications programme on elderly inpatients' competence to manage medications: a pilot study. 7 Clin Pharm Ther 2011;36:80-6, doi:10.1111/j.1365-2710.2009. 01157.x.

27. Fialová D, Onder G. Medication errors in elderly people: contributing factors and future perspectives. $\mathrm{Br} 7 \mathrm{Clin}$ Pharmacol 2009;67:641-5, doi:10.1111/j.1365-2125.2009. 03419.x.

28. Corona-Rojo JA, Altagracia-Martínez M, KravzovJinich J, et al. Potential prescription patterns and errors in elderly adult patients attending public primary health care centers in Mexico City. Clin Intervent Aging 2009;4: $343-50$.

29. Patanwala AE, Warholak TL, Sanders AB, et al. A prospective observational study of medication errors in a tertiary care emergency department. Ann Emerg Med 2010; 55:522-6, doi:10.1016/j.annemergmed.2009.12.017.

30. Fijn R, Van den Bemt PM, Chow M, et al. Hospital prescribing errors: epidemiological assessment of predictors. Br 7 Clin Pharmacol 2002;53:326-31, doi:10.1046/j.0306-5251. 2001.bjcp1558.doc.x.

31. Wakefield DS, Ward MM, Groath D, et al. Complexity of medication-related verbal orders. Am 7 Med Qual 2008;23:717, doi:10.1177/1062860607310922.

32. West DW, Levine S, Magram G, et al. Pediatric medication order error rates related to the mode of order transmission. Arch Pediatr Adolesc Med 1994;148:1322-6, doi:10.1001/ archpedi.1994.02170120084016. 


\section{Appendix. Details of prescription errors}

Situation

Type of error

Medication involved

72-year-old woman presenting to the ED after a fall and complaining of left ankle pain (CTAS 3); patient was admitted under Internal Medicine $\Rightarrow$ Total number of errors $=1$

1) $25 \mathrm{mg}$ was prescribed instead of patient's regular dose, $20 \mathrm{mg}$

Wrong dose

Wrong dose

Wrong dose

Wrong dose

Wrong dose

Wrong dose

Wrong dose

Wrong dose

Wrong dose

Wrong dose

Wrong dose

Wrong dose

Wrong dose

Wrong drug

Diltiazem ER/CD

14) Prescription did not mention extended release

67-year-old man sent to the ED for angiography and admission under palliative care (CTAS 3); patient was admitted under Medicine $\Rightarrow$ Total number of errors $=1$

15) Prescription did not mention extended release 


\section{Appendix. Continued}

\section{Situation}

Type of error

Medication involved

73-year-old man complaining of right hand numbness and weak fingers (CTAS 3); patient was discharged home $\Rightarrow$ Total number of errors $=1$

16) Prescription did not mention Diamicron MR

Wrong drug

72-year-old man complaining of weakness and dysuria, known for Crohn disease (CTAS 3); patient was admitted under Internal Medicine $\Rightarrow$ Total number of errors $=1$

17) Diamicron was prescribed instead Diamicron MR 81-year-old man presenting to the ED because of blood in colostomy bag (CTAS 3); patient was discharged home $\Rightarrow$ Total number of errors $=1$

18) Inappropriate indication considering patient condition 78-year-old man complaining of increased shortness of breath on exertion for 2-3 weeks (CTAS 2); patient was admitted under Cardiology $\Rightarrow$ Total number of errors $=1$

19) Medication was prescribed q.d. instead of t.i.d. 84-year-old woman presenting to the ED with diarrhea; patient was discharged home $\Rightarrow$ Total number of errors $=1$

20) Medication was prescribed b.i.d. instead of q.d.

90-year-old woman presenting with forehead and mouth trauma post fall (CTAS 3); patient was admitted under Family Medicine $\Rightarrow$ Total number of errors $=1$

21) Incorrect frequency; should not be as needed 83-year-old man presenting to the ED with respiratory distress (CTAS 2); patient was admitted under Internal Medicine $\Rightarrow$ Total number of errors $=1$

22) Medication was prescribed b.i.d. instead of q.d.

79-year-old woman complaining of chest pain (CTAS 3); patient was discharged home $\Rightarrow$ Total number of errors $=1$

23) Medication was prescribed q.4.h. instead of q.i.d.

91-year-old woman complaining of suprapubic pain (CTAS 3); patient was discharged $\Rightarrow$ Total number of errors $=1$

24) 10,000 IU was prescribed q.d. instead of q.week 91-year-old woman complaining of pelvic pain (CTAS 4); patient was admitted under Palliative Care (same patient as above, different visit) $\Rightarrow$ Total number of errors $=1$

25) Medication was prescribed q.d. instead of q.week 80-year-old woman complaining of right facial numbness (CTAS 3); patient was discharged home $\Rightarrow$ Total number of errors $=1$

26) Medication was prescribed q.d. instead of b.i.d.

80-year-old woman complaining of dizziness and shortness of breath (CTAS 2); patient was discharged home $\Rightarrow$ Total number of errors $=1$

27) Medication was prescribed $100 \mathrm{mg}$ q.d. instead of $50 \mathrm{mg}$ b.i.d.

93-year-old woman complaining of shortness of breath (CTAS 4); patient was discharged home $\Rightarrow$ Total number of errors $=1$

28) Prescription did not mention the dose 95-year-old man presenting to the ED with chest pain (CTAS 3); patient was admitted under Medicine $\Rightarrow$ Total number of errors $=1$

29) Prescription did not mention the dose

75-year-old woman presenting to the ED with shortness of breath with cough and green secretions (CTAS 3); patient was discharged home $\Rightarrow$ Total number of errors $=1$

30) Prescription did not mention the dose

Wrong drug

Wrong drug

Gliclazide (Diamicron MR)

Metformin

Wrong frequency

Wrong frequency

Wrong frequency

Wrong frequency

Wrong frequency

Wrong frequency

Wrong frequency

Wrong frequency

Fluticasone-salmeterol (Advair)

Cal D (Calcium and Vitamin D supplement)

Verapamil

Carbidopa-levodopa (Sinemet)

Vitamin D

Risedronate
Budesonide-formoterol (Turbuhaler)

Incomplete prescription Rivastigmine patch

Incomplete prescription Fluticasone (Flovent)

Incomplete prescription Irbesartan (Avapro) 


\section{Appendix. Continued}

Situation

Type of error

Medication involved

80-year-old man with fall, laceration, and bleeding on back of head patient on warfarin (CTAS 4); patient was discharged home $\Rightarrow$ Total number of errors $=1$

31) Prescription did not mention the dose

81-year-old woman who fell (CTAS 2); patient was admitted under Medicine $\Rightarrow$ Total number of errors $=1$

32) Prescription did not mention the dose

73-year-old man complaining of dysuria and lower abdominal pain

(CTAS 3); patient was discharged home $\Rightarrow$ Total number of

errors $=1$

33) Prescription did not mention the dose

64-year-old woman presenting with slurred speech and LOC, ICTAS

$2)$; patient was admitted under Cardiology $\Rightarrow$ Total number of errors $=1$

34) Prescription did not mention the frequency

99-year-old woman who fell (CTAS 3); patient was discharged $\Rightarrow$

Total number of errors $=1$

35) Prescription did not mention the frequency

82-year-old woman complaining of chest pain (CTAS 3); admitted under Surgery $\Rightarrow$ Total number of errors $=1$

36) Prescription did not mention the frequency

96-year-old woman presenting to the ED with shortness of breath and cyanosis (CTAS 2); patient was admitted under Cardiology $\Rightarrow$

Total number of errors $=1$

37) Prescription did not mention the route

86-year-old woman presenting to the ED with rectal bleeding (CTAS

3); patient was admitted to medical short-stay unit under Medicine

$\Rightarrow$ Total number of errors $=1$

38) Duration of treatment was not indicated on prescription

94-year-old woman presenting to the ED because of rib pain (CTAS 3); patient died in ED $\Rightarrow$ Total number of errors $=1$

39) Prescription mentioned only " 0.5 " instead of $0.5 \%$ Timoptic XE 84-year-old man complaining of shortness of breath exacerbation (CTAS 2); patient was admitted under Cardiology $\Rightarrow$ Total number of errors $=1$

40) Prescription did not mention the dose or frequency

88-year-old woman presenting to the ED for right knee pain post fall (CTAS 3); patient was discharged home $\Rightarrow$ Total number of errors $=1$

41) Prescription did not mention dose or frequency

83-year-old man complaining of hemoptysis (CTAS 3); patient was admitted under Internal Medicine $\Rightarrow$ Total number of errors $=1$

42) Prescription did not mention the dose or frequency

96-year-old woman with syncope (CTAS 3); patient was admitted under Family Medicine $\Rightarrow$ Total number of errors $=1$

43) Prescription did not mention any dose, route, or frequency 67-year-old woman sent by day hospital for PICC line insertion (CTAS 4); patient was admitted under Internal Medicine $\Rightarrow$ Total number of errors $=1$

44) Prescription did not mention the route or concentration 36-year-old man presenting with nausea and vomiting, abdominal pain, and diarrhea (CTAS 3); patient was discharged home $\Rightarrow$ Total number of errors $=1$

45) Prescription not signed by MD

54-year-old woman presenting to the ED because of weakness (CTAS 3); patient was discharged home $\Rightarrow$ Total number of errors $=1$

Incomplete prescription

Fluticasone-salmeterol (Advair)

Incomplete prescription

Travoprost

Incomplete prescription

Carbidopa-levodopa (Sinemet)

Incomplete prescription

Clotrimazole (Canesten)

Incomplete prescription

Furosemide (Lasix)

Incomplete prescription

Nitroglycerin patch

Incomplete prescription

Morphine

Incomplete prescription

Pantoprazole (Pantoloc)

Incomplete prescription

Timolol

Incomplete prescription

Vita-3 (multivitamins)

Incomplete prescription

Mirtazapine

Incomplete prescription

Latanoprost

Incomplete prescription

LMWH protocol

Incomplete prescription Latanoprost

Other: no signature 


\section{Appendix. Continued}

Situation
46) Prescription not signed by MD
94-year-old man with cancer presenting with general deterioration
(CTAS 3); patient was admitted under Medicine $\Rightarrow$ Total number of
errors $=1$
47) Prescription not signed by MD

74-year-old woman complaining of sore throat (CTAS 3); patient was admitted under Family Medicine $\Rightarrow$ Total number of errors $=1$

48) Prescription not signed by MD

90-year-old man complaining of shortness of breath (CTAS 2); patient was discharged home $\Rightarrow$ Total number of errors $=1$

49) Medication not available in hospital

85-year-old man complaining of vomiting (CTAS 3); patient was admitted under Surgery $\Rightarrow$ Total number of errors $=1$

50) Medication formulation not available in hospital

74-year-old man with a gait disturbance (CTAS 2); patient was admitted under Family Medicine

51) Medication not available in hospital

81-year-old woman complaining of shortness of breath and cough (CTAS 2); patient was discharged home $\Rightarrow$ Total number of errors $=1$

52) Patient is already taking ipratropium (Atrovent)

81-year-old man complaining of chest pain (CTAS 2); patient was admitted under Cardiology $\Rightarrow$ Total number of errors $=1$

53) Patient is already taking antacid (Maalox)

58-year-old woman complaining of general weakness post diarrhea (CTAS 2); patient was discharged home $\Rightarrow$ Total number of errors $=1$

54) Patient is already taking pantoprazole (Pantoloc)

80-year-old woman presenting to the ED with altered mental status $\times 1$ week (CTAS 2); patient was discharged home $\Rightarrow$ Total number of errors $=1$

55) Patient is already taking dorzolamide-timolol (Cosopt)

71-year-old man sent to the ED by MD office to rule out PE (CTAS 2); patient was admitted to the medical short-stay unit under Medicine $\Rightarrow$ Total number of errors $=1$

56) Patient has already been prescribed prednisone

71-year-old man complaining of sore throat (CTAS 3); patient was admitted to the medical short-stay unit under medicine $\Rightarrow$ Total number of errors $=1$

57) Prescription was illegible

86-year-old woman complaining of hip pain (CTAS 4); patient was sent to rehabilitation $\Rightarrow$ Total number of errors $=1$

58) Prescription was illegible

30-year-old woman complaining of nausea and vomiting (CTAS 3); admitted to the short-stay unit under Medicine $\Rightarrow$ Total number of errors $=1$

60) Prescription states to decrease drip to 2 units per hour and to stop drip

\section{Type of error}

Medication involved

Other: no signature

Other: no signature

Other: no signature

Other: nonformulary Sameterol xinafoate (Serevent Diskus) medication

Other: nonformulary

Levodopa-carbidopa (Levocarb) medication

Other: nonformulary medication

Forza (multivitamins)

Other: redundant

Tiotropium bromide (Spiriva) medication

Other: redundant

Antacid (Diovol) medication

Other: redundant medication

Omeprazole

Other: redundant

Timolol medication

Other: redundant Methylprednisolone medication

Other: illegible writing

Vancomycin

Other: illegible writing

Folate

Other: conflicting orders Insulin human recombinant (Humulin R) 


\section{Appendix. Continued}

65-year-old man complaining of left-sided chest pain and shortness of breath (CTAS 2); patient was admitted under Cardiology $\Rightarrow$ Total number of errors $=1$

59) Prescription time too early when angiogram was not scheduled yet

67-year-old man complaining of weakness and dizziness (CTAS 3): patient was discharged home $\Rightarrow$ Total number of errors $=2$

61) $10 \mathrm{mg}$ was prescribed instead of $80 \mathrm{mg}$

62) $10 \mathrm{mg}$ was prescribed instead of $0.5 \mathrm{mg}$

83-year-old man presenting to the ED because of abnormal blood test (CTAS 3); patient was discharged home $\Rightarrow$ Total number of errors $=2$

63) Wrong dose of medication was prescribed

64) Wrong dose of medication was prescribed

72-year-old man presenting to the ED with deterioration and confusion, urinary incontinence, and chills (CTAS 3); patient was admitted to the medical short-stay unit under Medicine $\Rightarrow$ Total number of errors $=2$

65) $1.5 \mathrm{mg}$ was prescribed; medication does not exist in this format

66) $1.5 \mathrm{mg}$ was represcribed by another MD; medication does not exist in this format

68-year-old man complaining of hematuria (CTAS 3); patient was discharged home $\Rightarrow$ Total number of errors $=2$

67) $180 \mathrm{mg}$ QD was prescribed instead of $240 \mathrm{mg}$ q.d.

68) Prescription did not mention extended release

88-year-old man presenting to the ED with increased shortness of breath and confusion (CTAS 2); patient was admitted under Internal Medicine $\Rightarrow$ Total number of errors $=2$

69) Medication was prescribed $120 \mathrm{mg}$ instead of $300 \mathrm{mg}$

70) Prescription did not mention the dose

82-year-old man presenting to the ED with sudden onset of shortness of breath (CTAS 2); patient was discharged home $\Rightarrow$ Total number of errors $=2$

71) $400 \mathrm{mg}$ was prescribed instead of $1,200 \mathrm{mg}$

72) Medication was prescribed PO instead of IV

82-year-old man complaining of pain behind the head, nausea and vomiting (CTAS 3); patient was admitted under Internal Medicine $\Rightarrow$ Total number of errors $=2$

73) Wrong dose was prescribed

74) Prescription did not mention the duration

79-year-old man sent to the ED for dialysis with increased shortness of breath (CTAS 2); patient was admitted under Family Medicine $\Rightarrow$ Total number of errors $=2$

75) Medication prescribed q.d.; q.d. is incorrect for renal failure indication

76) Prescription did not mention the frequency

86-year-old man with hyperkalemia who refuses to take his meds (CTAS 3); patient was discharged home $\Rightarrow$ Total number of errors $=2$

77) Prescription did not mention the dose

78) Prescription did not mention the frequency

60-year-old woman complaining of left-sided heaviness and weakness (CTAS 2); patient was admitted under Internal Medicine $\Rightarrow$ Total number of errors $=2$

79) Prescription did not mention the dose
Other: medication prescribed at the wrong time
Wrong dose

Wrong dose

Wrong dose

Wrong dose

Wrong dose

Wrong dose

Wrong dose

Wrong drug

Wrong dose

Incomplete prescription

\section{Wrong dose}

Wrong route

\section{Wrong dose \\ Incomplete prescription}

Wrong frequency

Incomplete prescription

Incomplete prescription Incomplete prescription
Acetylcysteine (Mucomyst)

Telmisartan (Micardis)

Dutasteride (Avodart)

Metformin

Dramamine

Olanzapine

Olanzapine

Diltiazem

Diltiazem ER/CD

Diltiazem

Tiotropium bromide (Spiriva)

Acetylcysteine (Mucomyst) Acetylcysteine (Mucomyst)

Amiodarone

Amiodarone

Levofloxacin (Levaquin)

Albuterol (Ventolin)

Oral iron (Palafer)

Betamethasone

Incomplete prescription Fluticasone (Flovent) 


\section{Appendix. Continued}

Situation
80) Both medications were prescribed at the same time
61-year-old man presenting to the ED with loss of consciousness
and hypotension (CTAS 2); patient was discharged home $\Rightarrow$ Total

number of errors $=3$

81) Wrong dose was prescribed

82) Wrong dose was prescribed

83) Medication was prescribed q.d. instead of q.4h.

79-year-old woman not responding to stimulus since the day before, was brought to the ED (CTAS 1); patient was admitted under Internal Medicine $\Rightarrow$ Total number of errors $=3$

84) Medication does not exist in the prescribed format

85) Timentin was prescribed despite a documented allergy to penicillin

86) Timentin was prescribed despite a documented allergy to penicillin (different prescription)

77-year-old man complaining of shortness of breath and weakness (CTAS 3); patient was admitted to the medical short-stay unit under Medicine $\Rightarrow$ Total number of errors $=3$

87) Medication was spelled incorrectly

88) Medication was prescribed b.i.d. instead of t.i.d.

89) Medication was prescribed T.i.d. instead of b.i.d.

74-year-old woman presenting with right hip pain (CTAS 3); patient was discharged home $\Rightarrow$ Total number of errors $=3$

90) Septra was prescribed instead of Septra DS

91) Medication prescribed t.i.d. instead of q.am

92) Patient is on medication but omitted from prescription

71-year-old woman sent to the ED by MD office for psychiatric evaluation (CTAS 3); patient was admitted under Psychiatry $\Rightarrow$ Total number of errors $=3$

93) Medication was prescribed q.h.s. instead of q.i.d.

94) Prescription did not mention the route or frequency

95) Prescription did not mention the route or frequency

42-year-old woman sent to the ED by MD office for psychiatric evaluation $(C T A S=N / A)$; patient was discharged home $\Rightarrow$ Total number of errors $=4$

96) $225 \mathrm{mg}$ was prescribed instead of $262.5 \mathrm{mg}$

97) $375 \mathrm{mg}$ was prescribed instead of $250 \mathrm{mg}$

98) Pravastatin was prescribed in $E D$, but patient is on fenofibrate (Lipidil) and not Pravastatin

99) Prescription did not mention the route

\author{
Wrong dose \\ Wrong dose \\ Wrong frequency
}

\section{Wrong dose \\ Wrong drug \\ Wrong drug}

\section{Wrong drug \\ Wrong frequency \\ Wrong frequency}

Wrong drug
Wrong frequency
Incomplete prescription

Entacapone (Comtan)

Carbidopa-levodopa (Sinemet)

Entacapone (Comtan)

Bupropion (Zyban)

Ticarcillin-clavulanate (Timentin)

Ticarcillin-clavulanate (Timentin)

Pilocarpine (Isopto Carpine)

Pilocarpine (Isopto Carpine)

Dorzolamide (Cosopt)

Trimethoprim-sulfamethoxazole (Septra) Celecoxib (Celebrex)

Calcium + vitamin D (Carbocal D)

$\mathrm{CD}=; \mathrm{CTAS}=$ Canadian Triage and Acuity Scale; ED = emergency department; IV = intravenous; LMWH = low-molecular-weight heparin; LOC = loss of consciousness; MD = physician; $\mathrm{MR}=$ modified release; $\mathrm{PE}=$ pulmonary embolism; $\mathrm{PICC}=$ peripherally inserted central catheter; $\mathrm{PO}=$ oral; $\mathrm{XR}=$ extended release. 